\title{
Neuroimaging: just a collection of brain image files?
}

\author{
Daniela Seixas $^{1,2,3 *}$ and Margarida Ayres Basto ${ }^{3}$ \\ 1 Faculty of Medicine of Porto University, Institute of Histology and Embryology, Porto, Portugal \\ 2 Instituto de Biologia Molecular e Celular, Universidade do Porto, Porto, Portugal \\ 3 Neuroradiology Department, São João Hospital, Porto, Portugal \\ *Correspondence: dseixas@med.up.pt
}

\section{INTRODUCTION}

With the advent of magnetic resonance imaging (MRI), and in particular in the 1990s with the introduction of functional MRI (fMRI) - a neuroimaging tool that measures hemodynamic responses related to neural activity - much has been done for the protection of the human subject. As imagers, we worry since the early days about magnet safety, and in every MRI suite there are in place several measures to protect individuals from physical injury, like screening for metal implants or the use of hearing protection (Shellock and Spinazzi, 2008). MRIsafe pacemakers are being developed (Mitka, 2009), while the European Union is trying to set a directive limiting the exposure to electromagnetic fields even of MRI personnel. There are though many other issues, concerning brain imaging both for clinical and research purposes, that have been much less or much later explored (Seixas and Ayres Basto, 2008), namely recruitment of vulnerable groups, informed consent, incidental findings, limitations of the techniques, interpretation and validity of results, confidentiality and privacy, applications outside the hospital or laboratory, or public communication of research results or imaging applications.

\section{INFORMED CONSENT, INCIDENTAL FINDINGS, IMAGE USE AND CONFIDENTIALITY}

Taking informed consent before brain imaging investigations is standard practice in the research and in the clinical settings. This issue however has been of more debate in the research environment, particularly when healthy subjects are involved (Illes et al., 2002; Grossman and Bernat, 2004). Informed consent is designed to protect both the volunteer and the researcher/research institution, in insuring that the subject understands the purpose of the brain MRI, that in case of an incidental finding there are standards to deal with these abnormalities, and that there are procedures to ensure confidentiality and privacy.
Incidental findings can be defined as suspicious anatomical or functional abnormalities that are unrelated to the purpose of the scan. Most authors agree that guidelines for detecting and communicating incidental abnormalities on brain MRI research are needed (Illes et al., 2004), but not without controversy. For example, Mamourian (2004), reviewing all research brain scans in his institution, reports how a brain aneurism was also found in his own scan for research purposes, and how successful the surgical treatment was. Conversely, what if the surgical procedure were to have severe complications in an otherwise asymptomatic person? Other authors argue that is neither feasible nor ethically required that every research scan be examined by a qualified specialist (Kirschen et al., 2006). A recent review on incidental findings on brain MRI performed either for research purposes or for clinical, occupational, or commercial screening, concluded that they are common and deserve to be mentioned when obtaining informed consent for brain MRI in research and clinical practice (Morris et al., 2009).

Brain images of patients/healthy individuals must be processed and archived, protecting privacy and allowing for retrieval. Privacy protection can be usually guaranteed if the scans are obtained as part of a medical procedure or in neuroscience dedicated laboratories. In a broader perspective, worldwide institutions are making an effort to organize and coordinate the extraordinary collections of brain structures and functional images that are being generated everyday. For example, in many clinical care facilities picture archiving and communications systems (PACS) are in use, which are computers dedicated to the storage, retrieval, distribution and presentation of medical images. Some centres are developing software that is able to create anonymised image teaching files from the PACS, either for local use or for World Wide Web-based applications (Rosset et al., 2002). This raises questions on subject consent in the context of secondary use of data. Can patients or research volunteers provide valid consent to unspecified future use of their brain images (Downie and Hadskis, 2005)?

\section{VULNERABLE GROUPS}

Imaging vulnerable groups is a sensitive topic for all parties involved, and these include children, elderly people, and cognitively or psychologically impaired patients. Questions of competence to consent arise, and safety, risks and logistics during MRI scanning may be particular of cognitively and/or emotionally impaired individuals. For example, Rosen et al. (2002) points out that patients with memory disorders may need to be closely monitored since they are vulnerable to disorientation; difficulties also occur with patients that have neurological deficits, in fMRI studies for pre-surgical planning of brain lesion removal, because of their reduced ability to perform the required motor, visual or language tasks during the scanning procedure.

Researchers must be careful, particularly in the field of neuropsychiatric disorders like schizophrenia or Alzheimer's disease, not to motivate further marginalisation or stigma, and to ponder the possible devastating consequences of false-positive and/or false-negative findings that may hinder diagnosis. Also on the research perspective, because of the constraints of working with neuropsychiatric populations, researchers tend to recruit less affected patients, leading to a selection bias (Illes et al., 2006).

\section{EXPECTATIONS AND MOTIVATIONS OF PARTICIPANTS}

There are few problems involving directly the research subject/patient not so popular in the literature that may, as well, conduct to data biasing: participants' expectations about neuroimaging experiments, their motivations to volunteer, and their perception of the overall scanning experience. 
Shaw et al. (2008) found that neuroimaging experiment subjects can become anxious prior to scanning and lacked a frame of reference to contextualise their expectations. In a 2007 study, the experiences of and attitudes toward fMRI/magnetoencephalography (MEG) were investigated. The findings were generally positive: all participants thought fMRI and MEG were safe techniques, most would recommend participating in imaging research to their friends and family, and they were also positive about participating in future brain imaging research (Cooke et al., 2007). Steinke (2004), in a non-imaging investigation in the clinical setting, studied the relationship between research ethics, informed consent, and participant recruitment. Key concerns identified by the author were the education level of participants, the relationship between participants and health care providers and illness severity (Steinke, 2004). Some of the issues that were raised are common to research practice, but others are clearly distinct. In neuroimaging experimental studies it is common to recruit human subjects from easy-to-recruit undergraduate student populations, or even from within the personnel of the institution conducting the experiment (where people maintain relationships and are sometimes accustomed to be participants). These samples are probably, at least for some research questions, not representative of the general population.

Revolving again around the incidental findings problem, Kirschen et al. (2006) investigated subjects' expectations in neuroimaging research. Their exploratory study revealed that although participants did provide written consent to a scanning procedure for research purposes alone, most of them still expected pathology to be detected and communicated to them (Kirschen et al., 2006). This study gives away an important hint: do research subjects/patients have their own objectives when volunteering for brain imaging studies? And are their motivations different if they belong to one group or the other?

From observation alone, it seems to be easier to recruit patients for neuroimaging research than normal controls. An example would be a bed-ridden patient from our practice, with severe pain, that volunteered for an experiment that included a particularly long brain scan. When questioned about why he volunteered, if doing so would be so costly to him, he answered that of course he wanted to help in the advance of knowledge about his disease, but mainly that he volunteered to try to convince his family and carers that his pain was not imaginary. Zijlmans et al. (2008), in a study of patients referred and then rejected for epilepsy surgery, concluded that most patients, once rejected, would like to benefit from novel techniques. But the patients' motivations are not clearly understood; is this because they want hope from another possible treatment, or because they want to comply with their doctor's willingness to try a new technique? We can also attempt to elaborate on the motivations of the healthy volunteer to participate in imaging studies, possibly distinct from the patient in the clinical environment. Do individuals volunteer just for the sake of science?Curiosity? For the compensation money? To return a favour to a colleague researcher? Or maybe to have a free brain scan done for those long-time disturbing headaches?

\section{CONCLUSION}

As radiologists or scientists making use of neuroimaging methods, we seem to worry much about certain issues that implicate the participants. Some of these concerns are important, like subjects' safety, and others are more in vogue, possibly the question of incidental findings. Image archiving and further use of brain images are being dealt with, both in the clinical and research practice, because they will allow rational use of resources and enhance knowledge of the brain by taking advantage of increased numbers. Some other issues still seem to be forgotten, namely the selection bias in difficult groups, and the individual motivations and expectations of subjects that may have an impact in the end result of an investigation or even in patient outcome. Taken together research participants' possible "hidden agenda" and their positive attitude towards neuroimaging experiences, as demonstrated by Cooke et al. (2007), should we not know more about their motivations to volunteer for brain imaging studies? And should we not plan ahead the impact of secondary use of imaging data? More research on our research is needed.

\section{REFERENCES}

Cooke, R., Peel, E., Shaw, R. L., and Senior, C. (2007). The neuroimaging research process from the participants' perspective. Int. J. Psychophysiol. 63, 152-158.
Downie, J., and Hadskis, M. (2005). Finding the right compass for issue-mapping in neuroimaging. Am. J. Bioeth. 5, 27-29.

Grossman, R. I., and Bernat, J. L. (2004). Incidental research imaging findings: Pandora's costly box. Neurology 62, 849-850.

Illes, J., Desmond, J. E., Huang, L. F., Raffin, T. A., and Atlas, S. W. (2002). Ethical and practical considerations in managing incidental findings in functional magnetic resonance imaging. Brain Cogn. 50, 358-365.

Illes, J., De Vries, R., Cho, M. K., and SchraedleyDesmond, P. (2006). ELSI priorities for brain imaging. Am. J. Bioeth. 6, 24-31.

Illes, J., Kirschen, M., Karetsky, K., Kelly, M., Saha, A., Desmond, J., Raffin, T., Glover, G., and Atlas, S. (2004) Discovery and disclosure of incidental findings in neuroimaging research. J. Magn. Reson. Imaging 20, 743-747.

Kirschen, M. P., Jaworska, A., and Illes, J. (2006). Subjects' expectations in neuroimaging research. J. Magn. Reson. Imaging 23, 205-209.

Mamourian, A. (2004). Incidental findings on research functional MR images: should we look? AJNR Am. J. Neuroradiol. 25, 520-522.

Mitka, M. (2009). Researchers seek MRI-safe pacemakers. JAMA 301, 476.

Morris, Z., Whiteley, W. N., Longstreth, W. T., Jr., Weber, F., Lee, Y. C., Tsushima, Y., Alphs, H., Ladd, S. C., Warlow, C., Wardlaw, J. M., and Al-Shahi Salman, R. (2009). Incidental findings on brain magnetic resonance imaging: systematic review and metaanalysis. BMJ 339, b3016.

Rosen, A. C., Bodke, A. L., Pearl, A., and Yeasavage, J. A. (2002). Ethical, and practical issues in applying functional imaging to the clinical management of Alzheimer's disease. Brain Cogn. 50, 498-519.

Rosset, A., Ratib, B., Geissbuhler, A., and Vallée, J.P. (2002). Integration of a multimedia teaching and reference database in a PACS environment. Radiographics 22, 1567-1677.

Seixas, D., and Ayres Basto, M. (2008). Ethics in fMRI studies: a review of the EMBASE and MEDLINE literature. Clin. Neuroradiol. 18, 79-87.

Shaw, R. L., Senior, C., Peel, E., Cooke, R., and Donnelly, L. S. (2008). Ethical issues in neuroimaging health research: an IPA study with research participants. J. Health Psychol. 13, 1051-1059.

Shellock, F. G., and Spinazzi, A. (2008). MRI safety update 2008: Part 2, screening patients for MRI. AJR Am. J. Roentgenol. 191, 1140-1149.

Steinke, E.E. (2004). Research ethics, informed consent, and participant recruitment. Clin. Nurse Spec. 18, 88-95.

Zijlmans, M., Buskens, E., Hersevoort, M., Huiskamp, G., van Huffelen, A. C., and Leijten, F. S. (2008). Should we reconsider epilepsy surgery? The motivation of patients once rejected. Seizure 17, 374-377.

Received: 04 September 2009; published online: 23 December 2009.

Citation: Front. Hum. Neurosci. (2009) 3:47. doi: 10.3389/neuro.09.047.2009

Copyright (C) 2009 Seixas and Ayres Basto. This is an open-access article subject to an exclusive license agreement between the authors and the Frontiers Research Foundation, which permits unrestricted use, distribution, and reproduction in any medium, provided the original authors and source are credited. 